\title{
A life support-based comprehensive treatment regimen dramatically lowers the in-hospital mortality of patients with fulminant myocarditis: a multiple center study
}

\author{
Sheng $\mathrm{Li}^{1 \dagger}$, Shengyong $\mathrm{Xu}^{2 \dagger}$, Chenze $\mathrm{Li}^{1 \dagger}$, Xiao Ran ${ }^{1}$, Guanglin Cui ${ }^{1}$, Mengying $\mathrm{He}^{1}$, \\ Kun Miao ${ }^{1}$, Chunxia Zhao ${ }^{1}$, Jiangtao Yan ${ }^{1}$, Rutai Hui ${ }^{3}$, Ning Zhou ${ }^{1}$, Yan Wang ${ }^{1}$, \\ Jiangang Jiang $^{1^{*}}$, Jing Zhang ${ }^{4^{*}} \&$ Daowen Wang ${ }^{1 *}$ \\ ${ }^{1}$ Division of Cardiology, Department of Internal Medicine, Tongji Hospital, Tongji Medical College, Huazhong University of Science and \\ Technology; Hubei Key Laboratory of Genetics and Molecular Mechanisms of Cardiological Disorders, Wuhan 430030, China; \\ ${ }^{2}$ Emergency Department of Union Hospital, Peking Union Medical College, Chinese Academy of Medical Sciences, Beijing 100037, China; \\ ${ }^{3}$ Fuwai Hospital, National Center for Cardiovascular Diseases, Chinese Academy of Medical Sciences, Peking Union Medical College, Beijing \\ 100037, China; \\ ${ }^{4}$ Fuwai Huazhong Cardiovascular Hospital, Zhengzhou 451450, China
}

Received December 21, 2018; accepted January 22, 2019; published online February 28, 2019

\begin{abstract}
Fulminant myocarditis (FM) has unacceptable high mortality. This study aimed to evaluate the therapeutic efficacy of a life support-based comprehensive treatment regimen (LSBCTR), a completely novel treatment regimen, for FM. A total of 169 FM patients recruited from January 2008 to December 2018 were divided into two groups: patients receiving LSBCTR (81 cases), which includes (i) mechanical life support (positive pressure respiration, intra-aortic balloon pump with or without extracorporeal membrane oxygenation), (ii) immunomodulation therapy using sufficient doses of glucocorticoids and immunoglobulins, and (iii) application of neuraminidase inhibitors, and those receiving conventional treatment (88 cases). The endpoints were in-hospital death and heart-transplantation. Of all the population, 44 patients $(26.0 \%)$ died in hospitals. Inhospital mortality was 3.7\% (3/81) for LSBCTR group and 46.6\% (41/88) for traditional treatment $(P<0.001)$. Early application of LSBCTR, mechanical life support, neuraminidase inhibitors, and immunomodulation therapy significantly contributed to reduction in in-hospital mortality. This study describes a novel treatment regimen for FM patients that dramatically reduces inhospital mortality. Its generalization and clinical application will efficiently save lives although further optimization is needed. This study offers an insight that virus infection induced inflammatory waterfall results in cardiac injury and cardiogenic shock and is the therapeutic target.
\end{abstract}

fulminant myocarditis, in-hospital mortality, life support-based comprehensive treatment regimen

Citation: Li, S., Xu, S., Li, C., Ran, X., Cui, G., He, M., Miao, K., Zhao, C., Yan, J., Hui, R., et al. (2019). A life support-based comprehensive treatment regimen dramatically lowers the in-hospital mortality of patients with fulminant myocarditis: a multiple center study. Sci China Life Sci $62,369-380$. https://doi.org/10.1007/s11427-018-9501-9

$\dagger$ Contributed equally to this work

*Corresponding authors (Daowen Wang, email: dwwang@tjh.tjmu.edu.cn; Jing Zhang, email: 1542682601@qq.com; Jiangang Jiang, email: jiangjg618@126.com) 


\section{INTRODUCTION}

Acute myocarditis is an acute myocardial inflammatory disorder characterized by various etiologies and clinical manifestations(Caforio et al., 2013; Caforio et al., 2017; Fung et al., 2016; Lazaros et al., 2017; Pollack et al., 2015). Fulminant myocarditis is a special class and the most severe form of acute myocarditis, characterized by rapid onset and progression, resulting in heart failure, serious hemodynamic compromise, hypotension, and cardiogenic shock (Ginsberg and Parrillo, 2013; Gupta et al., 2008; Maisch et al., 2014). Fulminant myocarditis is usually life-threatening, and its inhospital mortality rate is considerably high (up to $40 \%-$ $60 \%$ ), even when managed with aggressive pharmacological therapy and mechanical support (Diddle et al., 2015; Rodríguez et al., 2012; Ting et al., 2016). However, patients with fulminant myocarditis were reported to have much better long-term survival rates than patients with acute myocarditis if they live through the acute phase and if left ventricular function recovers completely within 1 month (McCarthy et al., 2000). Therefore, helping patients survive the acute stage is the greatest challenge for in-hospital management.

Acute myocardial infarction and myocarditis are the two most common reasons for acute heart failure and cardiogenic shock, which lead to pump dysfunction. The significant advances in acute coronary syndrome management have not been replicated for myocarditis. Early management has changed slightly over the decades despite substantial efforts. To date, there are no specific guidelines for the diagnosis and management of fulminant myocarditis except of our expert consensus (Section of Precision Medical of Chinese Society of Cardiology of Chinese Medical Association et al., 2017; Wang et al., 2019). Fulminant myocarditis has varied etiologies, including hypersensitivity to drugs, autoimmune disease, and drug toxicity; however, viral infections such as H1N1, enterovirus B, influenza, and parvovirus are major causes.

In recent years, considerable efforts have been made to save patients with fulminant myocarditis. Many sporadic case reports have suggested that mechanical support may be helpful (Ting et al., 2016), and some have indicated that large doses of glucocorticoids successfully saved some children with fulminant myocarditis (Bjelakovic et al., 2016; Chen et al., 2013). Continuous blood hemodialysis has been successfully used to treat severe sepsis patients by removing cytokine storms that cause inflammatory (Hirasawa et al., 2012). However, wide and fierce controversy and substantial concerns have existed for several decades on the rationality of glucocorticoid application as well as immunoglobulin for viral myocarditis as it enhances virus replication, and on the efficacy of intravenous injection of immunoglobulins; therefore, these reagents were restricted or even prohibited in clinical treatment practice. As a result, almost all patients with fulminant myocarditis underwent traditional treatments or "stepwise therapy" including vasoactive drug, especially dopamine, noradrenaline, and other inotropic agents for shock, and only when patients do not respond to these agents that mechanical life support such as extracorporeal membrane oxygenation (ECMO) is considered rather than early use, at least before the Chinese Society of Cardiology Expert Consensus Statement on the Diagnosis and Treatment of Adult Fulminant Myocarditis was published (Section of Precision Medical of Chinese Society of Cardiology of Chinese Medical Association et al., 2017; Wang et al., 2019). Thus, high in-hospital mortality is determined.

This study aimed to investigate a life support-based comprehensive treatment regimen (LSBCTR) based on the recommendations of this consensus statement and its therapeutic efficiency for adult fulminant myocarditis.

\section{RESULTS}

\section{Baseline characteristics}

From January 2008 to December 2018, a total of 169 patients who presented with fulminant myocarditis were included in our study. The average age of all enrolled patients was 34.00 (interquartile range (IQR), 26.00-48.00) years, and $49.7 \%$ of patients were women. The median time from onset of symptoms to hospitalization was 4 days, and the interquartile range was 3.00-7.00 days. Furthermore, $45.8 \%$ of patients presented with dyspnea when admitted to the hospital, $28.3 \%$ presented with chest pain, $79.5 \%$ presented with chest distress, $28.3 \%$ presented with palpitations, and $12.7 \%$ presented with syncope. The peak levels of cardiac troponin I, NT-pro-BNP, alanine aminotransferase, aspartate transaminase, blood urea nitrogen, creatinine, and the lowest levels of ejection fraction were recorded to represent the degree of organ injury during hospitalization.

Patients were compared with regard to whether they were treated with conventional (stepwise) method or LSBCTR. Results indicated that patients in the conventional group had a more severe cardiac, liver and renal dysfunction than did those in the LSBCTR group, which might be caused by inappropriate stepwise regimen during hospitalization. Nevertheless, a 1:1 propensity score-matching analysis was performed to match the baseline data between groups. All comparisons of baseline characteristics showed no statistical difference between the matched LSBCTR group and conventional group (Table 1). In addition, the characteristics of patients administered each treatment are described in Table 2.

\section{Efficacy and in-hospital mortalit}

Of the 169 patients, 44 patients $(26.0 \%)$ died in the hospital 
Table 1 Baseline characteristic and clinical outcomes of patients with fulminant myocarditis ${ }^{\text {a) }}$

\begin{tabular}{|c|c|c|c|c|c|c|c|c|}
\hline & \multicolumn{3}{|c|}{ Total population } & \multirow[b]{2}{*}{$P$} & \multicolumn{3}{|c|}{ Propensity-matched population } & \multirow[b]{2}{*}{$P$} \\
\hline & $\begin{array}{l}\text { Overall } \\
(n=169)\end{array}$ & $\begin{array}{l}\text { Conventional } \\
\text { group }(n=88)\end{array}$ & $\begin{array}{l}\text { LSBCTR group } \\
\qquad(n=81)\end{array}$ & & $\begin{array}{l}\text { Overall } \\
(n=76)\end{array}$ & $\begin{array}{l}\text { Conventional } \\
\text { group }(n=38)\end{array}$ & $\begin{array}{l}\text { LSBCTR group } \\
\qquad(n=38)\end{array}$ & \\
\hline \multicolumn{9}{|c|}{ Demographic and clinical characteristics } \\
\hline Age, mean (sd), year & $\begin{array}{c}34.00 \\
{[26.00,48.00]}\end{array}$ & $\begin{array}{c}35.00 \\
{[27.50,48.00]}\end{array}$ & $\begin{array}{c}33.00 \\
{[24.00,47.00]}\end{array}$ & 0.401 & $\begin{array}{c}37.50 \\
{[28.75,50.50]}\end{array}$ & $\begin{array}{c}37.00 \\
{[30.00,48.75]}\end{array}$ & $\begin{array}{c}39.00 \\
{[23.00,53.50]}\end{array}$ & 0.983 \\
\hline Female sex, $n(\%)$ & $84(49.7)$ & $39(44.3)$ & $45(55.6)$ & 0.144 & $39(51.3)$ & $19(50.0)$ & $20(52.6)$ & 0.818 \\
\hline $\begin{array}{c}\text { Time from symptoms to } \\
\text { hospitalization, median } \\
{[\mathrm{IQR}], \mathrm{d}}\end{array}$ & $4.00[3.00,7.00]$ & $4.00[2.00,7.00]$ & $4.00[3.00,7.00]$ & 0.716 & $4.00[2.00,7.00]$ & $4.00[2.00,7.00]$ & $4.00[2.00,7.00]$ & 0.983 \\
\hline Dyspnea, $n(\%)$ & $76(45.8)$ & $37(42.5)$ & $39(49.4)$ & 0.377 & $37(48.7)$ & $20(52.6)$ & $17(44.7)$ & 0.491 \\
\hline Chest pain, $n(\%)$ & $47(28.3)$ & $20(23.0)$ & $27(34.2)$ & 0.11 & $24(31.6)$ & $11(28.9)$ & $13(34.2)$ & 0.622 \\
\hline Chest distress, $n(\%)$ & $132(79.5)$ & $65(74.7)$ & $67(84.8)$ & 0.107 & $62(81.6)$ & $31(81.6)$ & $31(81.6)$ & 1 \\
\hline Palpation, $n(\%)$ & $47(28.3)$ & $28(32.2)$ & $19(24.1)$ & 0.245 & $26(34.2)$ & $14(36.8)$ & $12(31.6)$ & 0.629 \\
\hline Syncope, $n(\%)$ & $21(12.7)$ & $11(12.8)$ & $10(12.7)$ & 0.98 & $9(11.8)$ & $5(13.2)$ & $4(10.5)$ & 0.723 \\
\hline The peak cTnI, $n(\%)$ & & & & 0.181 & & & & 0.221 \\
\hline$<12000 \mathrm{pg} \mathrm{mL}^{-1}$ & $53(33.1)$ & $32(38.6)$ & $21(27.3)$ & & $22(28.9)$ & $8(21.1)$ & $14(36.8)$ & \\
\hline $12,000-30,000 \mathrm{pg} \mathrm{mL}^{-1}$ & $54(33.8)$ & $23(27.7)$ & $31(40.3)$ & & $26(34.2)$ & $16(42.1)$ & $10(26.3)$ & \\
\hline$>30,000 \mathrm{pg} \mathrm{mL}^{-1}$ & $53(33.1)$ & $28(33.7)$ & $25(32.5)$ & & $28(36.8)$ & $14(36.8)$ & $14(36.8)$ & \\
\hline $\begin{array}{c}\text { The peak NT-proBNP, } n \\
(\%)\end{array}$ & & & & 0.004 & & & & 0.952 \\
\hline$<6,000 \mathrm{pg} \mathrm{mL}^{-1}$ & $47(30.5)$ & $23(29.1)$ & $24(32.0)$ & & $25(32.9)$ & $13(34.2)$ & $12(31.6)$ & \\
\hline $6,000-20,000 \mathrm{pg} \mathrm{mL}^{-1}$ & $62(40.3)$ & $24(30.4)$ & $38(50.7)$ & & $34(44.7)$ & $17(44.7)$ & $17(44.7)$ & \\
\hline$>20,000 \mathrm{pg} \mathrm{mL}^{-1}$ & $45(29.2)$ & $32(40.5)$ & $13(17.3)$ & & $17(22.4)$ & $8(21.1)$ & $9(23.7)$ & \\
\hline The peak ALT, $n(\%)$ & & & & 0.063 & & & & 0.493 \\
\hline$<70 \mathrm{U} \mathrm{L}^{-1}$ & $51(31.1)$ & $20(23.3)$ & $31(39.7)$ & & $30(39.5)$ & $15(39.5)$ & $15(39.5)$ & \\
\hline $70-400 \mathrm{U} \mathrm{L}^{-1}$ & $54(32.9)$ & $30(34.9)$ & $24(30.8)$ & & $26(34.2)$ & 15 (39.5) & $11(28.9)$ & \\
\hline$>400 \mathrm{U} \mathrm{L}^{-1}$ & $59(36.0)$ & $36(41.9)$ & $23(29.5)$ & & $20(26.3)$ & $8(21.1)$ & $12(31.6)$ & \\
\hline The peak AST, $n(\%)$ & & & & 0.08 & & & & 0.583 \\
\hline$<120 \mathrm{U} \mathrm{L}^{-1}$ & $51(33.1)$ & $24(30.0)$ & $27(36.5)$ & & $29(38.2)$ & $14(36.8)$ & $15(39.5)$ & \\
\hline $120-600 \mathrm{U} \mathrm{L}^{-1}$ & $52(33.8)$ & $23(28.7)$ & $29(39.2)$ & & $28(36.8)$ & $16(42.1)$ & $12(31.6)$ & \\
\hline$>600 \mathrm{U} \mathrm{L}^{-1}$ & $51(33.1)$ & $33(41.2)$ & $18(24.3)$ & & $19(25.0)$ & $8(21.1)$ & $11(28.9)$ & \\
\hline The peak BUN, $n(\%)$ & & & & 0.112 & & & & 0.595 \\
\hline$<6 \mathrm{mmol} \mathrm{L}^{-1}$ & $45(29.8)$ & $18(23.1)$ & $27(37.0)$ & & $24(31.6)$ & $10(26.3)$ & $14(36.8)$ & \\
\hline $6-12 \mathrm{mmol} \mathrm{L}^{-1}$ & $52(34.4)$ & $27(34.6)$ & $25(34.2)$ & & $27(35.5)$ & $15(39.5)$ & $12(31.6)$ & \\
\hline$>12 \mathrm{mmol} \mathrm{L}^{-1}$ & $54(35.8)$ & $33(42.3)$ & $21(28.8)$ & & $25(32.9)$ & $13(34.2)$ & $12(31.6)$ & \\
\hline The peak Cre, $n(\%)$ & & & & 0.079 & & & & 1 \\
\hline$<70 \mu \mathrm{mol} \mathrm{L}^{-1}$ & $49(30.1)$ & $23(27.1)$ & $26(33.3)$ & & $28(36.8)$ & $14(36.8)$ & $14(36.8)$ & \\
\hline $70-120 \mu \mathrm{mol} \mathrm{L}^{-1}$ & $58(35.6)$ & $26(30.6)$ & $32(41.0)$ & & $30(39.5)$ & $15(39.5)$ & $15(39.5)$ & \\
\hline$>120 \mu \mathrm{mol} \mathrm{L}^{-1}$ & $56(34.4)$ & $36(42.4)$ & $20(25.6)$ & & 18 (23.7) & $9(23.7)$ & $9(23.7)$ & \\
\hline The low EF, $n(\%)$ & & & & 0.929 & & & & 0.765 \\
\hline$>40 \%$ & $44(28.2)$ & $23(28.7)$ & $21(27.6)$ & & $18(23.7)$ & $8(21.1)$ & $10(26.3)$ & \\
\hline $25 \%-40 \%$ & $65(41.7)$ & $34(42.5)$ & $31(40.8)$ & & $33(43.4)$ & $18(47.4)$ & $15(39.5)$ & \\
\hline$<25 \%$ & $47(30.1)$ & $23(28.7)$ & $24(31.6)$ & & $25(32.9)$ & $12(31.6)$ & $13(34.2)$ & \\
\hline \multicolumn{9}{|l|}{ Endpoints } \\
\hline In-hospital death, $n(\%)$ & $44(26.0)$ & $41(46.6)$ & $3(3.7)$ & $<0.001$ & $17(22.4)$ & $16(42.1)$ & $1(2.6)$ & $<0.001$ \\
\hline Survivors & & & & & - & - & - & - \\
\hline $\begin{array}{l}\text { Length of hospital stay for } \\
\text { survivors, median [IQR], d }\end{array}$ & $\begin{array}{c}15.00 \\
{[11.00,20.00]}\end{array}$ & $\begin{array}{c}17.00 \\
{[14.00,21.50]}\end{array}$ & $\begin{array}{c}13.00 \\
{[11.00,18.00]}\end{array}$ & 0.004 & - & - & - & - \\
\hline
\end{tabular}

a) IQR, interquartile range; cTnI, cardiac troponin I; NT-proBNP, N-terminal Pro-B-type natriuretic peptide; ALT, alanine aminotransferase; AST, aspartate transaminase; BUN, blood urea nitrogen; Cre, creatinine; EF, ejection fraction; LSBCT, life support based comprehensive treatment regimen. 
Table 2 Baseline characteristics and clinical outcomes in each treatment ${ }^{\mathrm{a})}$

\begin{tabular}{|c|c|c|c|c|c|c|c|c|c|c|c|c|}
\hline & \multicolumn{2}{|c|}{ IABP or ECMO } & \multirow[b]{2}{*}{$P$} & \multicolumn{2}{|c|}{ Antiviral group } & \multirow[b]{2}{*}{$P$} & \multicolumn{2}{|c|}{ IVIG } & \multirow[b]{2}{*}{$P$} & \multicolumn{2}{|c|}{ Glucocorticoid } & \multirow[b]{2}{*}{$P$} \\
\hline & $\begin{array}{c}\text { No IABP or } \\
\text { ECMO } \\
\text { group } \\
(n=62)\end{array}$ & $\begin{array}{l}\text { IABP or } \\
\text { ECMO } \\
\text { group } \\
(n=107)\end{array}$ & & $\begin{array}{l}\text { No antiviral } \\
\text { group } \\
(n=49)\end{array}$ & $\begin{array}{l}\text { Antiviral group } \\
\qquad(n=120)\end{array}$ & & $\begin{array}{l}\text { No IVIG } \\
\text { group } \\
(n=50)\end{array}$ & $\begin{array}{l}\text { IVIG group } \\
\quad(n=119)\end{array}$ & & $\begin{array}{l}\text { No glucocorti- } \\
\text { coid group } \\
\quad(n=24)\end{array}$ & $\begin{array}{l}\text { Glucocorticoid } \\
\text { group } \\
(n=145)\end{array}$ & \\
\hline \multicolumn{13}{|c|}{ Demographic and clinical characteristics } \\
\hline Age, mean (sd), year ${ }^{3}$ & $\begin{array}{l}34.50[26.75,3 \\
\quad 47.00]\end{array}$ & $\begin{array}{l}33.00[24.00 \\
\quad 48.00]\end{array}$ & 0.62 & $\begin{array}{l}33.00[28.00 \\
\quad 48.00]\end{array}$ & $\begin{array}{c}35.00[24.00 \\
47.25]\end{array}$ & 0.88 & $\begin{array}{c}36.50[29.25 \\
48.00]\end{array}$ & $\begin{array}{l}33.00[24.00 \\
47.00]\end{array}$ & 0.314 & $\begin{array}{l}36.00[32.00 \\
\quad 50.75]\end{array}$ & $\begin{array}{c}34.00[24.00 \\
47.00]\end{array}$ & 0.151 \\
\hline Female sex, $n(\%)$ & $26(41.9)$ & $58(54.2)$ & 0.124 & $17(34.7)$ & $67(55.8)$ & 0.013 & $22(44.0)$ & $62(52.1)$ & 0.336 & $9(37.5)$ & $75(51.7)$ & 0.197 \\
\hline $\begin{array}{l}\text { Time from symptoms } \\
\text { to hospitalization, } \\
\text { median [IQR], d }\end{array}$ & $\begin{array}{l}4.00[2.00 \\
7.00]\end{array}$ & $\begin{array}{c}4.00[3.00 \\
7.00]\end{array}$ & 0.44 & $\begin{array}{c}5.00[2.00 \\
7.00]\end{array}$ & $\begin{array}{c}4.00[3.00 \\
7.00]\end{array}$ & 0.469 & $\begin{array}{c}5.00[2.00 \\
7.00]\end{array}$ & $\begin{array}{c}4.00[3.00 \\
7.00]\end{array}$ & 0.431 & $\begin{array}{c}4.00[2.00 \\
7.00]\end{array}$ & $\begin{array}{c}4.00[3.00 \\
7.00]\end{array}$ & 0.63 \\
\hline Dyspnea, $n(\%)$ & $28(45.2)$ & $48(46.2)$ & 0.901 & $23(46.9)$ & $53(45.3)$ & 0.847 & $25(50.0)$ & $51(44.0)$ & 0.474 & $13(54.2)$ & $63(44.4)$ & 0.373 \\
\hline Chest pain, $n(\%)$ & $13(21.0)$ & $34(32.7)$ & 0.105 & $12(24.5)$ & $35(29.9)$ & 0.479 & $12(24.0)$ & $35(30.2)$ & 0.418 & $6(25.0)$ & $41(28.9)$ & 0.697 \\
\hline Chest distress, $n(\%)$ & 45 (72.6) & $87(83.7)$ & 0.087 & $37(75.5)$ & $95(81.2)$ & 0.408 & $37(74.0)$ & $95(81.9)$ & 0.247 & $17(70.8)$ & $115(81.0)$ & 0.254 \\
\hline Palpation, $n(\%)$ & $22(35.5)$ & $25(24.0)$ & 0.113 & $12(24.5)$ & $35(29.9)$ & 0.479 & $13(26.0)$ & $34(29.3)$ & 0.664 & $4(16.7)$ & $43(30.3)$ & 0.171 \\
\hline Syncope, $n(\%)$ & $8(13.1)$ & $13(12.5)$ & 0.909 & $3(6.1)$ & $18(15.5)$ & 0.098 & $5(10.2)$ & $16(13.8)$ & 0.527 & $3(12.5)$ & $18(12.8)$ & 0.971 \\
\hline \multicolumn{2}{|c|}{ The peak cTnI, $n(\%)$} & & 0.217 & & & 0.289 & & & 0.029 & & & 0.329 \\
\hline$<12,000 \mathrm{pg} \mathrm{mL}^{-1}$ & $23(39.0)$ & $30(29.7)$ & & $19(42.2)$ & $34(29.6)$ & & $22(48.9)$ & $31(27.0)$ & & $8(38.1)$ & $45(32.4)$ & \\
\hline $\begin{array}{c}12,000-30,000 \mathrm{pg} \\
\mathrm{mL}^{-1}\end{array}$ & $15(25.4)$ & $39(38.6)$ & & $14(31.1)$ & $40(34.8)$ & & $11(24.4)$ & $43(37.4)$ & & $9(42.9)$ & $45(32.4)$ & \\
\hline$>30,000 \mathrm{pg} \mathrm{mL}^{-1}$ & $21(35.6)$ & $32(31.7)$ & & $12(26.7)$ & $41(35.7)$ & & $12(26.7)$ & $41(35.7)$ & & $4(19.0)$ & $49(35.3)$ & \\
\hline \multicolumn{2}{|c|}{ The peak NT-proBNP, $n(\%)$} & & 0.153 & & & 0.215 & & & 0.026 & & & 0.594 \\
\hline$<6,000 \mathrm{pg} \mathrm{mL}^{-1}$ & $19(33.9)$ & $28(28.6)$ & & $11(25.6)$ & $36(32.4)$ & & $16(37.2)$ & $31(27.9)$ & & $5(23.8)$ & $42(31.6)$ & \\
\hline $6,000-20,000 \mathrm{pg} \mathrm{mL}^{-}$ & $17(30.4)$ & $45(45.9)$ & & $15(34.9)$ & $47(42.3)$ & & $10(23.3)$ & $52(46.8)$ & & $8(38.1)$ & $54(40.6)$ & \\
\hline$>20,000 \mathrm{pg} \mathrm{mL}^{-1}$ & $20(35.7)$ & $25(25.5)$ & & $17(39.5)$ & $28(25.2)$ & & $17(39.5)$ & $28(25.2)$ & & $8(38.1)$ & $37(27.8)$ & \\
\hline \multicolumn{2}{|c|}{ The peak ALT, $n(\%)$} & & 0.128 & & & 0.397 & & & 0.709 & & & 0.509 \\
\hline$<70 \mathrm{U} \mathrm{L}^{-1}$ & $15(24.2)$ & $36(35.3)$ & & $12(25.5)$ & $39(33.3)$ & & $13(26.5)$ & $38(33.0)$ & & $6(26.1)$ & $45(31.9)$ & \\
\hline $70-400 \mathrm{U} \mathrm{L}^{-1}$ & $26(41.9)$ & $28(27.5)$ & & $19(40.4)$ & $35(29.9)$ & & $17(34.7)$ & $37(32.2)$ & & $10(43.5)$ & $44(31.2)$ & \\
\hline$>400 \mathrm{U} \mathrm{L}^{-1}$ & $21(33.9)$ & $38(37.3)$ & & $16(34.0)$ & $43(36.8)$ & & $19(38.8)$ & $40(34.8)$ & & $7(30.4)$ & $52(36.9)$ & \\
\hline \multicolumn{2}{|c|}{ The peak AST, $n(\%)$} & & 0.648 & & & 0.858 & & & 0.17 & & & 0.255 \\
\hline$<120 \mathrm{U} \mathrm{L}^{-1}$ & $20(34.5)$ & $31(32.3)$ & & $15(36.6)$ & $36(31.9)$ & & $18(40.9)$ & $33(30.0)$ & & $9(50.0)$ & $42(30.9)$ & \\
\hline $120-600 \mathrm{U} \mathrm{L}^{-1}$ & $17(29.3)$ & $35(36.5)$ & & $13(31.7)$ & $39(34.5)$ & & $10(22.7)$ & $42(38.2)$ & & $4(22.2)$ & $48(35.3)$ & \\
\hline$>600 \mathrm{U} \mathrm{L}^{-1}$ & $21(36.2)$ & $30(31.2)$ & & $13(31.7)$ & $38(33.6)$ & & $16(36.4)$ & $35(31.8)$ & & $5(27.8)$ & $46(33.8)$ & \\
\hline \multicolumn{2}{|c|}{ The peak BUN, $n(\%)$} & & 0.989 & & & 0.154 & & & 0.079 & & & 0.08 \\
\hline$<6 \mathrm{mmol} \mathrm{L}^{-1}$ & $17(29.8)$ & $28(29.8)$ & & $11(26.8)$ & $34(30.9)$ & & $8(18.6)$ & $37(34.3)$ & & $3(17.6)$ & $42(31.3)$ & \\
\hline $6-12 \mathrm{mmol} \mathrm{L}^{-1}$ & $20(35.1)$ & $32(34.0)$ & & $19(46.3)$ & $33(30.0)$ & & $20(46.5)$ & $32(29.6)$ & & $10(58.8)$ & $42(31.3)$ & \\
\hline$>12 \mathrm{mmol} \mathrm{L}^{-1}$ & $20(35.1)$ & $34(36.2)$ & & $11(26.8)$ & $43(39.1)$ & & $15(34.9)$ & $39(36.1)$ & & $4(23.5)$ & $50(37.3)$ & \\
\hline \multicolumn{2}{|c|}{ The peak Cre, $n(\%)$} & & 0.883 & & & 0.084 & & & 0.328 & & & 0.07 \\
\hline$<70 \mu \mathrm{mol} \mathrm{L}{ }^{-1}$ & $20(32.3)$ & $29(28.7)$ & & $9(19.1)$ & $40(34.5)$ & & $11(22.4)$ & $38(33.3)$ & & $2(9.1)$ & $47(33.3)$ & \\
\hline $70-120 \mu \mathrm{mol} \mathrm{L}{ }^{-1}$ & $21(33.9)$ & $37(36.6)$ & & $22(46.8)$ & $36(31.0)$ & & $18(36.7)$ & $40(35.1)$ & & $10(45.5)$ & $48(34.0)$ & \\
\hline$>120 \mu \mathrm{mol} \mathrm{L}^{-1}$ & $21(33.9)$ & $35(34.7)$ & & $16(34.0)$ & $40(34.5)$ & & $20(40.8)$ & $36(31.6)$ & & $10(45.5)$ & $46(32.6)$ & \\
\hline The low EF, $n(\%)$ & & & 0.22 & & & 0.888 & & & 0.671 & & & 0.33 \\
\hline$>40 \%$ & $12(20.7)$ & $32(32.7)$ & & $13(29.5)$ & $31(27.7)$ & & $15(32.6)$ & $29(26.4)$ & & $7(36.8)$ & $37(27.0)$ & \\
\hline $25 \%-40 \%$ & $25(43.1)$ & $40(40.8)$ & & $19(43.2)$ & $46(41.1)$ & & $17(37.0)$ & $48(43.6)$ & & $9(47.4)$ & $56(40.9)$ & \\
\hline$<25 \%$ & $21(36.2)$ & $26(26.5)$ & & $12(27.3)$ & $35(31.2)$ & & $14(30.4)$ & $33(30.0)$ & & $3(15.8)$ & $44(32.1)$ & \\
\hline Endpoints & & & & $\begin{array}{c}12.00[3.00 \\
18.00]\end{array}$ & $\begin{array}{l}13.00[10.00 \\
18.00]\end{array}$ & 0.257 & $\begin{array}{c}12.00[3.25 \\
17.00]\end{array}$ & $\begin{array}{c}13.50[9.00 \\
18.75]\end{array}$ & 0.075 & & & \\
\hline $\begin{array}{c}\text { In-hospital death, } n \\
(\%)\end{array}$ & $24(38.7)$ & $20(18.7)$ & 0.004 & $19(38.8)$ & $25(20.8)$ & 0.016 & $19(38.0)$ & $25(21.0)$ & 0.022 & $9(37.5)$ & $35(24.1)$ & 0.167 \\
\hline \multicolumn{13}{|l|}{ Survivors } \\
\hline $\begin{array}{l}\text { Length of hospital } \\
\text { stay for survivors, } \\
\text { median [IQR], d }\end{array}$ & $\begin{array}{l}16.00[14.00 \\
20.75]\end{array}$ & $\begin{array}{l}14.50[11.00, \\
20.00]\end{array}$ & 0.19 & $\begin{array}{l}16.50[12.25 \\
20.00]\end{array}$ & $\begin{array}{c}14.50[11.00 \\
20.00]\end{array}$ & 0.246 & $\begin{array}{c}16.00[12.00 \\
18.50]\end{array}$ & $\begin{array}{l}15.00[11.00 \\
21.00]\end{array}$ & 0.66 & $\begin{array}{c}17.00[12.00 \\
19.50]\end{array}$ & $\begin{array}{l}15.00[11.00 \\
20.00]\end{array}$ & 0.65 \\
\hline
\end{tabular}

a) IQR, interquartile range; cTnI, cardiac troponin I; NT-proBNP, N-terminal Pro-B-type natriuretic peptide; ALT, alanine aminotransferase; AST, aspartate transaminase; BUN, blood urea nitrogen; Cre, creatinine; EF, ejection fraction. Glucocorticoid was defined as early-use of more than 200 mg methylprednisolo. 
and none received heart transplantation. Patients treated with LSBCTR had lower in-hospital mortality compared with those administered conventional treatment (3.7\% vs. $46.6 \%$; adjusted odds ratio (OR), $0.01 ; 95 \%$ confidence interval $(\mathrm{CI})$, $0.00-0.05 ; P<0.001$ ) (Table 1, Figure 1A). Among the survivors, LSBCTR obviously shortened the hospitalization time, compared with those in conventional group (13.00 days (11.00-18.00 days) vs. 17.00 days (14.00-21.50 days); $P=0.004)$. In the propensity-matched cohort, patients in the LSBCTR group were matched $1: 1$ to those in the conventional group. The results also indicated that patients treated with the LSBCTR had lower in-hospital mortality $(2.6 \%$ vs. $42.1 \%$, adjusted OR, 0.04; 95\% CI, $0.00-0.20 ; P=0.002$ ) (Table 1, Figure 1B).

\section{Attribution analysis of treatment effects}

To further explore the individual effects of treatment, we tested the relationship of the use of IABP, antiviral drugs, IVIG, and glucocorticoids with the prognosis. The results indicated that IABP or/and ECMO application, antiviral drug, and IVIG administrations significantly decrease the inhospital death rate individually (18.7\% vs. $38.7 \%$; adjusted OR, $0.17 ; 95 \%$ CI, $0.05-0.48 ; P=0.001$ for IABP or ECMO, $20.8 \%$ vs. $38.8 \%$; adjusted OR, 0.22; 95\% CI, 0.07-0.65; $P=0.008$ for antiviral drug; $21.0 \%$ vs. $38.0 \%$; adjusted $\mathrm{OR}$, $0.22 ; 95 \%$ CI, $0.07-0.65 ; P=0.008$ for IVIG) (Table 2, Figure $2)$. Additionally, the application of glucocorticoid showed an obvious trend of lower in-hospital death rate $(24.1 \%$ vs. $37.5 \%$; adjusted OR, 0.35 ; 95\% CI, $0.08-1.43 ; P=0.142$ ). These findings showed that an early combination of those methods and techniques contributes to clinical efficacy.

\section{Analysis of causes of death}

Analysis of causes of death showed that the prognosis of

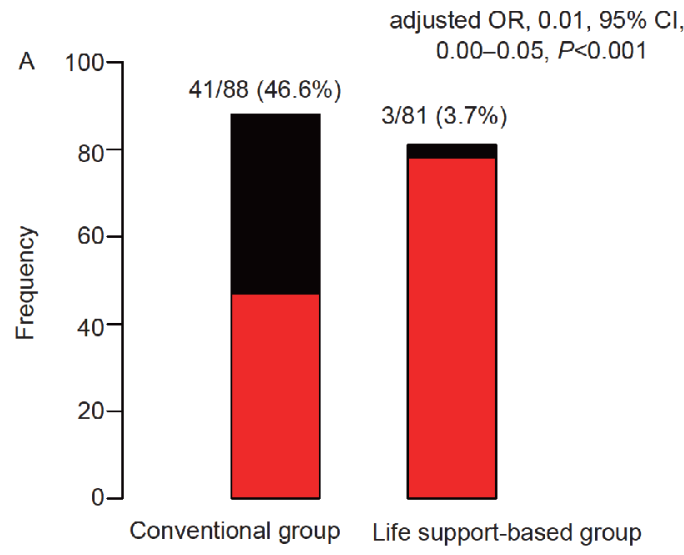

fulminant myocarditis was associated with the degree of organ injury. Patients with an NT-pro-BNP level higher than $20,000 \mathrm{pg} \mathrm{mL}^{-1}$ had a 23.52 times higher risk of in-hospital death than did those with an NT-pro-BNP level less than $6,000 \mathrm{pg} \mathrm{mL}^{-1}$. Liver insufficiency and renal insufficiency also suggested higher in-hospital mortality (Table 3). More importantly, long-term usage of large doses of noradrenaline for cardiogenic shock therapy rather than life support to reduce the heart load accelerated the death of patients (adjusted OR, 2.68; 95\% CI, 1.32-5.48; $P=0.007$ ) (Table 3), possibly by increasing peripheral vessel resistance and decreasing the supply to organs, especially the heart.

Although the survival rate was extremely high in the LSBCTR group (96.3\%), there were still three deaths in those groups. The cause analysis found that two patients died from disseminated intravascular coagulation that was not discovered in time and secondary bleeding because they received long-term noradrenaline and dopamine therapy (3 days) rather than the addition of ECMO when the IABP was used; however, it was not enough to provide proper circulatory support. One patient died because the cardiac function did not recover despite sufficient circulatory support achieved using the combination of IABP and ECMO.

\section{Glucocorticoid administration decreased animal mor- tality and inhibited virus replication in the heart}

The cardiac output, the E/A value and heart rate were significantly lower in the CVB3 group, compared with those in the control group. After administration of dexamethasone on CVB3 mice, the cardiac function markedly improved. The levels of cTnT were higher in the CVB3 group than in CVB3 + Dex group and control group (Table S1 in Supporting Information). Besides, myocardial fibrosis was detected in CVB3 group in the eighth day and dexamethasone could

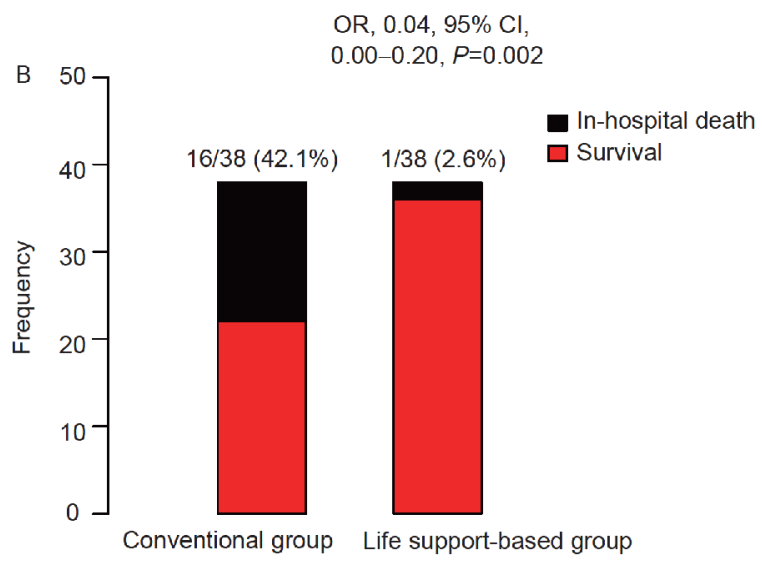

Figure 1 Comparisons between the conventional group and life support-based group. A, Of the patients who underwent conventional treatment ( $n=88)$, 41 died in the hospital. Of the patients who underwent life support-based treatment $(n=81)$, three died in the hospital. The adjusted odds ratio (OR) and $95 \%$ confidence interval (CI) were calculated by multivariable logistic regression. B, Of propensity-matched population who underwent conventional treatment $(n=38), 16$ patients died in the hospital. Of the patients who underwent life support-base treatment $(n=38)$, one died in the hospital. The OR and $95 \%$ CI were calculated by univariable logistic regression. 


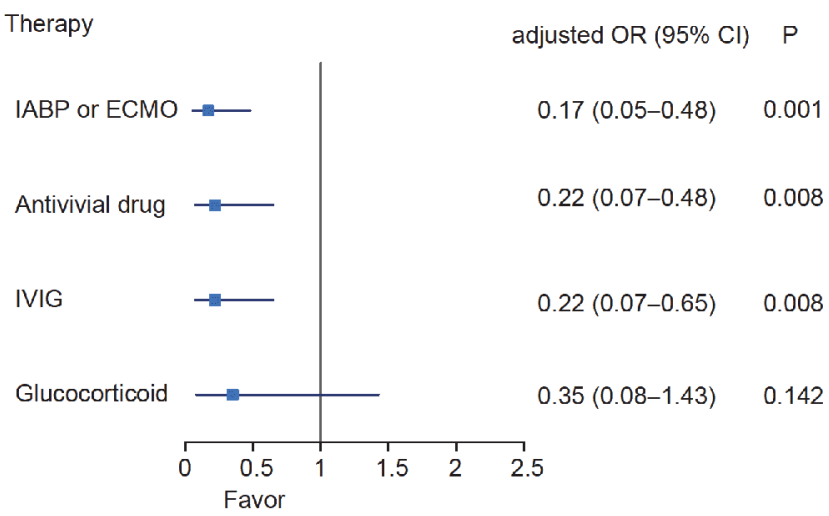

Figure 2 Odds ratio (OR) of in-hospital deaths for each treatment in the overall population. IABP, intra-aortic balloon pump; IVIG, intravenous immunoglobulin; CRRT, continuous renal replacement therapy. The OR and $95 \%$ confidence interval $(\mathrm{CI})$ were calculated by multivariable logistic regression.

significantly attenuate fibrosis (Figure S1 in Supporting Information). Experiments in Coxsackievirus infection-induced fulminant myocarditis showed that intraperitoneal injection of dexamethasone $0.75 \mathrm{mg} \mathrm{d}^{-1} \mathrm{~kg}^{-1}$ (corresponding to $200 \mathrm{mg} \mathrm{d}^{-1}$ of methylprednisolone in adult) for 3 days significantly reduced animal mortality, increased survival rate $(66.7 \%)$ compared with control (25\%) (Figure 3). CVB3 RNA copy number were 60.2 -fold $(P<0.05)$ lower in the heart of mice in CVB3+Dex group than in CVB3 group, respectively (copy number in CVB3 mice: $1.17 \times 10^{6}$ $\pm 3.70 \times 10^{5} \quad(n=8)$ versus CVB3+Dex mice $1.94 \times 10^{4}$ $\left.\pm 2.02 \times 10^{3}(n=8) ; P<0.05\right)$, indicating a lower viral replication in the myocardium of CVB3+Dex mice compared with CVB3 mice (Figure 4). These data suggest that glucocorticoid application markedly reduced mortality and viral replication in fulminant myocarditis animals.

\section{DISCUSSION}

This study investigated LSBCTR on the basis of the recommendations of the consensus statement and its therapeutic efficiency for adult fulminant myocarditis (Section of Precision Medical of Chinese Society of Cardiology of Chinese Medical Association et al., 2017; Wang et al., 2019). We found that application of this regimen significantly improved cardiac pump function and dramatically reduced inhospital mortality rates of this fatal disease from $46.6 \%$ to less than $5 \%$.

This study used LSBCTR for patients with fulminant myocarditis for the first time and demonstrated that this comprehensive regimen dramatically lowered in-hospital mortality of consecutive fulminant myocarditis patients and successfully saved lives without complications, compared with a stepwise conventional regimen. The key points of the LSBCTR include mechanical circulatory support and re-
March (2019) Vol.62 No.3

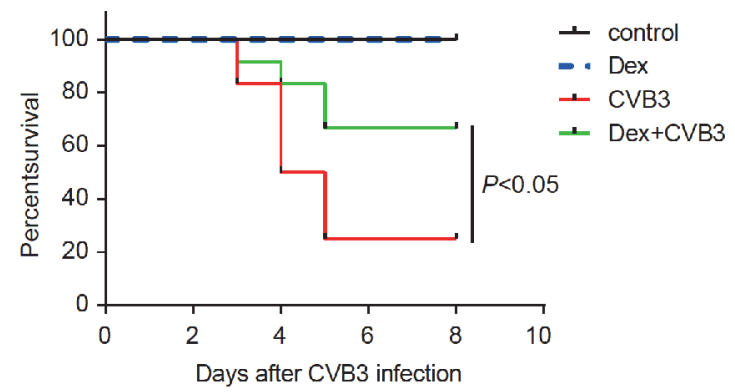

Figure 3 Impact of dexamethasone (Dex) on CVB3 infection in $\mathrm{A} / \mathrm{J}$ mice. A/J mice were infected with 105 PFU of CVB3 (Nancy), while dexamethasone treatment was carried out immediately after viral infection for 3 days. Proportional survival of animals during the 8 days of infection was implemented into a Kaplan-Meier survival curve (control $n=8$, Dex $n=8$, CVB3 $n=24$, CVB3+Dex $n=24$; log-rank test).

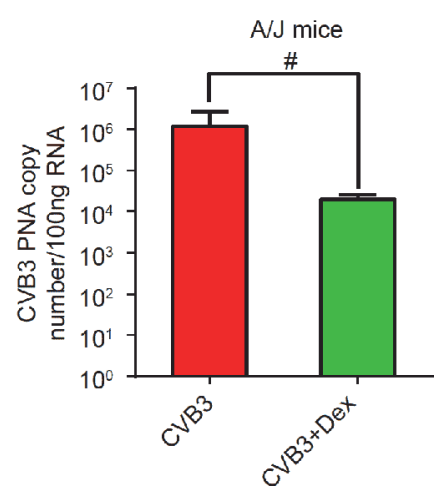

Figure 4 Viral burden of differently treated A/J mice. Animals were sacrificed at the 8 th day after CVB3 infection. Viral load was determined by real-time PCR detecting CVB3 RNA copy levels in total myocardial RNA (CVB3 $n=8$, CVB3+Dex $n=8$; Unpaired $t$-tests).

spiratory support to decrease cardiac load, immune modulation with sufficient doses of glucocorticoids and immunoglobulins, and antiviral therapy. We especially emphasize that combination therapy should be started as early as possible and avoid or reduce the need for vasoactive agents, especially adrenergic alpha receptor agonists such as noradrenaline.

Fulminant myocarditis is defined as inflammatory myocardial injury complicated by severe cardiac dysfunction manifested as acute heart failure and symptomatic hypotension or cardiogenic shock according to Chinese Expert Consensus Statement (Ginsberg and Parrillo, 2013; Gupta et al., 2008; Maisch et al., 2014; Section of Precision Medical of Chinese Society of Cardiology of Chinese Medical Association et al., 2017; Wang et al., 2019). The pathophysiology of fulminant myocarditis involves direct myocardial injury caused by pathogens, and the indirect injury is caused by the over immunologic response and overwhelmed secretion of inflammatory cytokines, resulting in an acute inflammatory waterfall effect and profound decrease of myocardial contractility, reduced cardiac output, and cardiogenic shock. Fulminant myocarditis has extremely high 
Table 3 Analysis of the cause of death by univariate logistic regression

\begin{tabular}{|c|c|c|c|}
\hline Variables & OR & $95 \% \mathrm{CI}$ & $P$ \\
\hline \multicolumn{4}{|l|}{ The peak NT-proBNP } \\
\hline$<6,000 \mathrm{pg} \mathrm{mL}^{-1}$ & & Ref & \\
\hline $6,000-20,000 \mathrm{pg} \mathrm{mL}^{-1}$ & 5.4 & $1.38-35.92$ & 0.033 \\
\hline$>20,000 \mathrm{pg} \mathrm{mL}^{-1}$ & 23.52 & $6.21-155.08$ & $<0.001$ \\
\hline \multicolumn{4}{|l|}{ The peak ALT } \\
\hline$<70 \mathrm{U} \mathrm{L}^{-1}$ & & Ref & \\
\hline $70-400 \mathrm{U} \mathrm{L}^{-1}$ & 2.38 & $0.86-7.31$ & 0.108 \\
\hline$>400 \mathrm{U} \mathrm{L}^{-1}$ & 4.79 & $1.86-14.12$ & 0.002 \\
\hline \multicolumn{4}{|l|}{ The peak AST } \\
\hline$<120 \mathrm{U} \mathrm{L}^{-1}$ & & Ref & \\
\hline $120-600 \mathrm{U} \mathrm{L}^{-1}$ & 2.76 & $0.94-9.30$ & 0.077 \\
\hline$>600 \mathrm{U} \mathrm{L}^{-1}$ & 7.56 & $2.76-24.58$ & $<0.001$ \\
\hline \multicolumn{4}{|l|}{ The peak BUN } \\
\hline$<6 \mathrm{mmol} \mathrm{L}^{-1}$ & & Ref & \\
\hline $6-12 \mathrm{mmol} \mathrm{L}^{-1}$ & 3.76 & $1.19-19.45$ & 0.039 \\
\hline$>12 \mathrm{mmol} \mathrm{L}^{-1}$ & 13.46 & $4.20-60.60$ & $<0.001$ \\
\hline \multicolumn{4}{|l|}{ The peak Cre } \\
\hline$<70 \mu \mathrm{mol} \mathrm{L}-1$ & & Ref & \\
\hline $70-120 \mu \mathrm{mol} \mathrm{L}^{-1}$ & 1.32 & $0.44-4.21$ & 0.628 \\
\hline$>120 \mu \mathrm{mol} \mathrm{L}^{-1}$ & 6.21 & $2.40-18.37$ & $<0.001$ \\
\hline \multicolumn{4}{|l|}{ The lowest EF } \\
\hline$>40 \%$ & & Ref & \\
\hline $25 \%-40 \%$ & 0.27 & $0.11-0.63$ & 0.003 \\
\hline$<25 \%$ & 0.16 & $0.05-0.43$ & $<0.001$ \\
\hline Noradrenaline & 2.68 & $1.32-5.48$ & 0.007 \\
\hline
\end{tabular}

a) NT-proBNP, N-terminal Pro-B-type natriuretic peptide; ALT, alanine aminotransferase; AST, aspartate transaminase; BUN, blood urea nitrogen; Cre, creatinine; $\mathrm{EF}$, ejection fraction.

in-hospital mortality, and there are no treatment regimens or guidelines. However, there are many case reports of successful treatment using different methods such as ECMO. Even if application of IABP or ECMO alone in some extent successfully saves some patients with fulminant myocarditis, its in-hospital mortality still is up to $50 \%$ (Diddle et al., 2015) or $70 \%$ (Rodríguez et al., 2012) or the patients need to receive heart transplantation, suggesting that a single or stepwise therapy strategy is not enough to save life.

The pathophysiological mechanism of fulminant myocarditis is different from those of ischemic heart disease and dilated cardiomyopathy; therefore, many general recommendations are not useful and are even harmful for fulminant myocarditis. For example, inotropic agents such as dopamine and dobutamine usually cause severe arrhythmias. In fulminant myocarditis, the injury is almost always diffusing, and primary and severe diffusing myocardial injuries induce acute pump failure. These underlying pathophysiologic differences indicate that different ways to treat fulminant myocarditis are needed.

The standard therapy for cardiogenic shock primarily consists of vasopressors and inotropic agents, such as catecholamines (norepinephrine, dopamine, and dobutamine), to enhance the vascular tone and force the heart to contract. For the stepped conventional treatment regimen, catecholamines were used first and patient responses were monitored. Mechanical circulatory support is considered only when the patient is unresponsive. Notably, the limited hemodynamic benefits of inotropes and vasopressors are counterbalanced by their adverse effects, such as potential myocardial injury, arrhythmia, and compromise of microcirculation. Moreover, rapid deterioration is often observed in patients with fulminant myocarditis. In the present study, long-term use of large doses of noradrenaline was proven to dramatically increase in-hospital mortality. Therefore, conventional or stepwise treatment strategy may lead to missing the best treatment window or irreversible outcomes. Based on the pathophysiological features of fulminant myocarditis, an LSBCTR is warranted and is likely the right choice at this time (Puymirat et al., 2017; Wang et al., 2019).

Our novel treatment regimen, the LSBCTR, is entirely different from the conventional regimen, as it is based on 
novel recognition and understanding of pathophysiology for fulminant myocarditis, i.e., over immunologic response and inflammatory waterfall leading to cardiac injury.

(i) Mechanical life support provides both circulatory and respiratory supports and importantly reduces cardiac work or let it rest: circulatory support is most important for patients with low refractory cardiac output. Our observations showed that immediate use of the IABP provides effective circulatory support for fulminant myocarditis patients in shock. For most of them, it can increase systolic blood pressure by over $20 \mathrm{mmHg}$ and simultaneously decrease heart rate by 20-30 bpm. In addition, the application of IABP also decreased the rate of in-hospital death. A randomized clinical trial of the IABP for fulminant myocarditis is lacking; however, sporadic reports have shown the value of the IABP for successful treatment (Okai et al., 2012; Ukimura et al., 2010; Ukimura et al., 2013). During the early stage, in a pilot study, we used the IABP as fundamental management during the therapeutic regimen because of its hemodynamic benefits of reducing the harm caused by vasopressors. The dose and duration of vasopressors were decreased significantly after the IABP was used. If an IABP is not sufficient for circulatory support, ECMO is added. Of the total population, 20 patients received ECMO support with IABP because of lower blood pressure (blood pressure approximately $70 / 50 \mathrm{mmHg}$ ). The combination of IABP and ECMO provided enough circulation support, and the blood pressure returned to approximately $95-100 / 70 \mathrm{mmHg}$. All patients recovered after 3-5 days of support. In addition, there is no potential cardiotoxicity or systemic microcirculation compromise. Moreover, the percutaneous insertion can be performed simply and conveniently in the cardiac catheter laboratory or at the bedside. ECMO, or more precisely extracorporeal life support, is another alternative method of maintaining systemic blood flow while avoiding possible cardiotoxicity of inotropes and vasopressors (Lim et al., 2017). Recently, there has been a trend of early use of extracorporeal life support to rescue severe cases. However, because of its cost and associated complication rates, extracorporeal life support is often considered the last resort to achieve hemodynamic stability, restore physiological milieu, and facilitate organ function recovery. Mechanical respiratory support is also an important part of this therapeutic regimen. It will correct hypoxemia, and more importantly, it can prevent and treat adult acute respiratory distress syndrome and reduce the cardiac load. Rodríguez et al. (Rodrigues et al., 2017) reported that $\sim 70 \%$ of patients with acute respiratory distress syndrome have cardiogenic shock and severe pneumonia and that only $40 \%$ have X-ray signs of acute respiratory distress syndrome, which requires mechanical respiratory support. Both mechanical respiratory support and circulatory support (life support) help reduce the cardiac work load and allow the failing heart to rest; there- fore, it should be routinely used as early as possible.

(ii) Immunomodulation therapy: an overactive immune response and inflammatory waterfall effects are considered fundamental mechanisms of fulminant myocarditis, which provide the rationale for the use of sufficient intravenous doses of both immunoglobulins and corticosteroids. IVIG was first tested for acute myocarditis in a pediatric population (Drucker et al., 1994). Data suggested that use of highdose IVIG $\left(2 \mathrm{mg} \mathrm{kg}^{-1}\right.$ over $\left.24 \mathrm{~h}\right)$ for the treatment of acute myocarditis is associated with improved recovery of left ventricular function and allows better survival compared to the non-IVIG group. A multicenter study showed that the use of high-dose IVIG (1-2 $\mathrm{g} \mathrm{kg}^{-1}$ over 2 days) for adults was associated with amelioration of the clinical course, increased left ventricular function, lower mortality rates, and marked reduction in inflammatory cytokines (Kishimoto et al., 2014). With fulminant myocarditis, high-dose IVIG improved left ventricular ejection fraction in six patients, and all patients survived (Goland et al., 2008). In a recent retrospective cohort study, Isogal et al. reported no significant association between IVIG use for fulminant myocarditis and in-hospital mortality (Isogai et al., 2015). However, the IVIG group included patients treated with various doses of IVIG, unlike the earlier randomized controlled trial in which patients in the IVIG group were treated with a predefined dose $\left(2 \mathrm{~g} \mathrm{~kg}^{-1}\right)$. In our study, patients were administered $20 \mathrm{~g}$ of immunoglobulin per day for 3-5 days in the IVIG group, unless there was quick stabilization during the first 3 days of our early combined regimen. Importantly, intact immunoglobulin administration markedly ameliorates fulminant myocarditis via $\mathrm{Fc}$ fragment-mediated antiinflammatory action and suppression of dendritic cellmediated initial antigen-priming process in myosin-induced rat fulminant myocarditis (Shioji et al., 2001), which provides the basis for immunoglobulin application in the treatment of fulminant myocarditis. The therapeutic effectiveness of glucocorticoids for fulminant myocarditis has remained controversial because it might facilitate viral replication. Nevertheless, high doses of intravenous methylprednisolone ( $1 \mathrm{~g} \mathrm{~d}^{-1}$ for 3 days) significantly improve the hemodynamic parameters of patients refractory to mechanical circulatory support (Nakashima et al., 2013), indicating the importance of targeting the overactive immune system with steroids. Interestingly, Nakamura et al. demonstrated that glucocorticoid administration after Coxsackievirus infection can reduce the mortality of fulminant myocarditis mice and tissue virus titers (Nakamura et al., 2013). Our preliminary data indicate that appropriate doses of corticosteroid do not increase virus replication, but decrease both virus titers and death in Coxsackievirus B3 infection-induced mouse model, although its mechanism needs further study. Additionally, acute administration of glucocorticoid can activate Akt and endothelial nitric oxidase to provide endothelial and cardiac 
protection (Hafezi-Moghadam et al., 2002). Thus, our clinical results also demonstrate that patients with fulminant myocarditis benefit greatly from sufficient doses of glucocorticoid application with mechanical life supports.

(iii) Administration of neuraminidase inhibitors. Rodríguez et al. (Rodríguez et al., 2012) reported that early use of the antiviral agent oseltamivir can reduce mortality rates of influenza patients; more recently, Zhang and colleagues (Zhang et al., 2018) demonstrated that neuraminidase-1 released from injured myocardium increases the plasma level of $\mathrm{N}$-acetylneuraminic acid, which aggravates cardiac damage, while neuraminidase inhibitors can reduce the damage. In this study, interestingly, we found that oseltamivir significantly contributed to the curative effect of our LSBCTR.

In conclusion, this study showed that the LSBCTR dramatically decreased the in-hospital mortality of fulminant myocarditis patients compared with the stepwise conventional treatment. It is worth popularizing because its clinical use will save numerous lives. Nevertheless, more studies are needed to figure out whether patients with FM should receive further treatment with small amount of steroid and last for how long time after discharge by follow-up of endocardial biopsy, magnetic imaging tests and blood inflammatory cytokines levels to determine the existence and level of inflammation, and by echocardiography for cardiac function, and to understand better mechanisms for immunomodulation therapy by basic research to optimize this treatment regimen.

\section{METHODS}

This study was funded by the National Natural Science Foundation of China. The executive committee designed and oversaw the conduct of the study and data analysis. All protocols and methods were approved by the respective local ethics committees and were conducted in accordance with principles of the Declaration of Helsinki and the International Conference on Harmonization Guidelines for Good Clinical Practice. We obtained written informed consent from the participants or their relatives.

The study was a multicenter, observational, and comparative study that investigated the therapeutic efficiency of a LSBCTR for adult fulminant myocarditis compared with conventional treatments at four medical centers in China (Tongji Hospital, Tongji Medical College, Huazhong University of Science and Technology, Wuhan, Hubei Province; Union Hospital and Fuwai Hospital, Peking Union Medical College, Chinese Academy of Medical Sciences, Beijing; Fuwai Huazhong Cardiovascular Hospital, Zhengzhou, Henan Province; Xiangya Hospital, Changsha, Hunan Province).

\section{Patient enrollment}

A total of 169 consecutive patients with fulminant myocarditis admitted to Tongji Hospital, Tongji Medical College, Huazhong University of Science and Technology, Hubei Province; Union Hospital and Fuwai Hospital, Peking Union Medical College, Chinese Academy of Medical Sciences, Beijing; Fuwai Huazhong Cardiovascular Hospital, Henan Province; Xiangya Hospital, Hunan Province between January 2008 and December 2018 were included. Data regarding illness history, laboratory tests, special tests, diagnosis, and treatment were extracted from the electronic medical records by two independent investigators. The diagnostic criteria for fulminant myocarditis were as follows: acute onset of symptoms of cardiac dysfunction such as dyspnea, palpitations, chest pain, and/or syncope with precursory symptom of viral infection; biomarkers of myocardial injury dramatically increased such as troponin I, B-type natriuretic peptide, or N-terminal pro-B-type natriuretic peptide (NTpro-BNP) levels; imaging for cardiac injury showing marked diffused reductions in left ventricular wall movement with dramatically decreased left ventricular ejection fraction $<45 \%$; and cardiogenic shock (e.g., systolic blood pressure $\leq 90 \mathrm{mmHg}$ or mean arterial pressure $<70 \mathrm{mmHg}$ or systolic blood pressure decrease $>40 \mathrm{mmHg}$ ) or hypotension, which is associated with signs of hypofusion (cyanosis, cold extremities, oliguria, and/or changes in mental status). The exclusion criteria of our study cohort were age younger than 11 years; possible acute coronary syndrome but unable to undergo coronary angiography to distinguish acute coronary syndrome from fulminant myocarditis; myocardial injury caused by sepsis, chemotherapeutical agents, or poison; and unstable hemodynamics or shock caused by hypovolemia.

\section{Therapy and assessment of clinical outcomes}

All 169 patients fulfilling the inclusion criteria were analyzed and classified according to their treatments. (i) Traditional therapy: patients were first treated with a stepped regimen of conventional medicines for heart failure and cardiogenic shock. Mechanical life support such as IABP or continuous renal replacement therapy was only successively added when hemodynamics was still unstable after titration and usage of high-dose vasopressors and inotropes. Antiviral medicines, intravenous immunoglobulin (IVIG), and steroids were used according to the physician's preference. (ii) LSBCTR according to the recommendations of China Consensus Statement (Section of Precision Medical of Chinese Society of Cardiology of Chinese Medical Association et al., 2017; Wang et al., 2019): all patients in this group received three classes of therapy: mechanical life support (artificial positive pressure respiration, intra-aortic balloon pump (IABP), if with or without ECMO), immunological mod- 
ulation therapy using sufficient doses of glucocorticoids and immunoglobulins and application of neuraminidase inhibitors. In brief, (A) all patients received immediate steroids (immediate intravenous injection of $20 \mathrm{mg}$ of dexamethasone followed by $200 \mathrm{mg}$ of methylprednisolone per day for 5 days, and weaned on the week thereafter; full-dose intravenous administration of immunoglobulin (10-20 $\mathrm{g} \mathrm{d}^{-1}$ for the first 3-5 days and then $10 \mathrm{~g} \mathrm{~d}^{-1}$ for the next 3-5 days); (B) mechanical life supports, including circulatory support and respiratory support; all patients received IABP, and if circulation or tissue perfusion was still not enough, ECMO was added. Vasopressors such as dopamine or norepinephrine were used only during the preparation periods for IABP or ECMO and titrated as little as needed to maintain systolic blood pressure at approximately $80 \mathrm{mmHg}$. Respiratory support was applied as needed (if patients had respiratory distress, decreased $\mathrm{SaO}_{2}$, or fast respiration rate $>20$ times $\min ^{-1}$ ). Duplex intermittent positive airway pressure was preferentially used for cooperative patients with tachypnea. If it was ineffective or not tolerated by the patient, then a tracheal cannula was used. (C) Immediate administration of neuraminidase inhibitor: all patients received oral oseltamivir (Tamiflu) (75 mg, twice a day) for 7-10 days. The endpoints were in-hospital death and heart transplantation.

\section{Effects of glucocorticoids on animal survival and virus titer in mouse model}

A previous study indicated that appropriate doses of glucocorticoids inhibited inflammation in the heart and reduced mortality in Coxsackievirus-induced myocarditis (Nakamura et al., 2013). To further provide beneficial support for glucocorticoids as treatment of fulminant myocarditis, we established a fulminant myocarditis mouse model by Coxsackievirus infection as described previously (Althof et al., 2018; Nakamura et al., 2013). Briefly, virus-free A/J (H2a) male mice received intraperitoneal injection of $1 \times 10^{5}$ PFU Coxsackievirus, and immediately after viral infection, the mice received dexamethasone $0.75 \mathrm{mg} \mathrm{kg}^{-1} \mathrm{~d}^{-1}$, i.p., for 3 days. Eight days later, the animals were sacrificed to test CVB3 RNA copy number in the heart as described previously (Tschope et al., 2017). CVB3 copy number was detected by real-time PCR using the forward primer 5'GTGGTGGGCTATGGAGTATG-3' and the reverse primer 5'-ATTGCACAGAGTCAAGGGT-3' at a final concentration of $50 \mathrm{ng} \mu \mathrm{L}^{-1}$. Total RNA was derived from heart tissues by TRIzol Reagent (Invitrogen, Carlsbad, CA, USA) according to the manufacturer's protocol. Total RNA was quantified using a Qubit 3 Fluorometer and CVB3 copy number in murine heart tissues was determined per $100 \mathrm{ng}$ RNA.

\section{Statistical analysis}

Continuous variables were presented as mean \pm standard deviation or median (interquartile range) depending on the data distribution. Categorical variables were presented as counts (percentage). For continuous variables with normal distribution, the independent $t$-test was used to test the differences between two groups. Otherwise, the Wilcoxon ranksum test was applied. Categorical variables were compared using the chi-square test. We performed multivariable logistical regression to identify independent associations of care patterns with clinical outcomes. Variables, including age, sex, time from symptoms to hospitalization, troponin I, NT-pro-BNP, alanine aminotransferase, aspartate transaminase, blood urea nitrogen, creatinine, and left ventricular ejection fraction were included in our multiple adjustments. To further enhance the robustness of our findings, additional propensity score-matching analyses were conducted. We estimated the propensity score using a logistic regression model with the variables listed above. Patients in the conventional treatment group were matched 1:1 to patients in the LSBCTR group based on their propensity score with nearest neighbor matching without replacement. The caliper value was set at 0.2 , and an absolute standardized difference less than $10 \%$ for a given covariate indicated a small imbalance.

Statistical analyses were performed using $\mathrm{R}$ version 3.1.4 (R Core Team, Vienna, Austria), and the sample size was calculated using $\mathrm{R}$ with the stats package. Estimated event rates were $60 \%$ for the conventional group and $10 \%$ for the LSBCTR group. To attain a statistical power of 0.90 with a 1:1 ratio between the two groups and a type I error rate of 0.05 , a total of 34 patients were needed. All comparisons were two-sided, and $P<0.05$ was considered statistically significant.

Compliance and ethics The author(s) declare that they have no conflict of interest. Clinical Trial Registration URL: http://www.clinicaltrials.gov. Unique identifier: NCT03268642.

Acknowledgements This work was supported by the National Basic Research Program of China (2012CB518004), the key project of the National Natural Science Foundation of China (81630010, 81790624), and National Key Research and Development Program of China (SQ2017YFSF090157).

\section{References}

Althof, N., Goetzke, C.C., Kespohl, M., Voss, K., Heuser, A., Pinkert, S., Kaya, Z., Klingel, K., and Beling, A. (2018). The immunoproteasomespecific inhibitor ONX 0914 reverses susceptibility to acute viral myocarditis. EMBO Mol Med 10, 200-218.

Bjelakovic, B., Vukomanovic, V., and Jovic, M. (2016). Fulminant myocarditis in children successfully treated with high dose of methylprednisolone. Ind J Pediatr 83, 268-269.

Caforio A.L., Pankuweit S., Arbustini E., Basso C., Gimeno-Blanes J., Felix S.B., Fu M., Helio T., Heymans S., Jahns R., et al. (2013). Current state of knowledge on aetiology, diagnosis, management, and therapy of myocarditis: a position statement of the European Society of Cardiology 
Working Group on Myocardial and Pericardial Diseases. Eur Heart J 34, 2636-2648, 2648a-2648d.

Caforio, A.L.P., Malipiero, G., Marcolongo, R., and Iliceto, S. (2017). Myocarditis: a clinical overview. Curr Cardiol Rep 19, 63.

Chen H.S., Wang W., Wu S.N., and Liu J.P. (2013). Corticosteroids for viral myocarditis. Cochrane Database Syst Rev 10, CD004471.

Diddle, J.W., Almodovar, M.C., Rajagopal, S.K., Rycus, P.T., and Thiagarajan, R.R. (2015). Extracorporeal membrane oxygenation for the support of adults with acute myocarditis. Crit Care Med 43, 10161025.

Drucker, N.A., Colan, S.D., Lewis, A.B., Beiser, A.S., Wessel, D.L., Takahashi, M., Baker, A.L., Perez-Atayde, A.R., and Newburger, J.W. (1994). Gamma-globulin treatment of acute myocarditis in the pediatric population.. Circulation 89, 252-257.

Fung, G., Luo, H., Qiu, Y., Yang, D., and McManus, B. (2016). Myocarditis. Circ Res 118, 496-514.

Ginsberg, F., and Parrillo, J.E. (2013). Fulminant myocarditis. Crit Care Clin 29, 465-483.

Goland, S., Czer, L.S.C., Siegel, R.J., Tabak, S., Jordan, S., Luthringer, D., Mirocha, J., Coleman, B., Kass, R.M., and Trento, A. (2008). Intravenous immunoglobulin treatment for acute fulminant inflammatory cardiomyopathy: series of six patients and review of literature. Can J Cardiol 24, 571-574.

Gupta, S., Markham, D.W., Drazner, M.H., and Mammen, P.P. (2008). Fulminant myocarditis. Nat Rev Cardiol 5, 693-706.

Hafezi-Moghadam, A., Simoncini, T., Yang, Z., Limbourg, F.P., Plumier, J. C., Rebsamen, M.C., Hsieh, C.M., Chui, D.S., Thomas, K.L., Prorock, A.J., et al. (2002). Acute cardiovascular protective effects of corticosteroids are mediated by non-transcriptional activation of endothelial nitric oxide synthase. Nat Med 8, 473-479.

Hirasawa, H., Oda, S., Nakamura, M., Watanabe, E., Shiga, H., and Matsuda, K. (2012). Continuous hemodiafiltration with a cytokineadsorbing hemofilter for sepsis. Blood Purif 34, 164-170.

Isogai, T., Yasunaga, H., Matsui, H., Tanaka, H., Horiguchi, H., and Fushimi, K. (2015). Effect of intravenous immunoglobulin for fulminant myocarditis on in-hospital mortality: propensity score analyses. J Cardiac Failure 21, 391-397.

Kishimoto, C., Shioji, K., Hashimoto, T., Nonogi, H., Lee, J.D., Kato, S., Hiramitsu, S., and Morimoto, S. (2014). Therapy with immunoglobulin in patients with acute myocarditis and cardiomyopathy: analysis of leukocyte balance. Heart Vessels 29, 336-342.

Lazaros, G., Oikonomou, E., and Tousoulis, D. (2017). Established and novel treatment options in acute myocarditis, with or without heart failure. Expert Rev Cardiovasc Ther 15, 25-34.

Lim, H.S., Howell, N., and Ranasinghe, A. (2017). Extracorporeal life support: physiological concepts and clinical outcomes. J Cardiac Failure 23, 181-196.

Maisch, B., Ruppert, V., and Pankuweit, S. (2014). Management of fulminant myocarditis: a diagnosis in search of its etiology but with therapeutic options. Curr Heart Fail Rep 11, 166-177.

McCarthy, R.E., Boehmer, J.P., Hruban, R.H., Hutchins, G.M., Kasper, E. K., Hare, J.M., and Baughman, K.L. (2000). Long-term outcome of fulminant myocarditis as compared with acute (nonfulminant) myocarditis. N Engl J Med 342, 690-695.

Nakamura, H., Kunitsugu, I., Fukuda, K., Matsuzaki, M., and Sano, M. (2013). Diverse stage-dependent effects of glucocorticoids in a murine model of viral myocarditis. J Cardiol 61, 237-242.

Nakashima, H., Umeyama, Y., and Minami, K. (2013). Successive immunosuppressive treatment of fulminant myocarditis that is refractory to mechanical circulatory support. Am J Case Rep 14,
$116-119$.

Okai, I., Inoue, K., Maruyama, M., Maruyama, S., Komatsu, K., Nishizawa, H., Okazaki, S., Fujiwara, Y., Sumiyoshi, M., and Daida, H. (2012). Transbrachial intra-aortic balloon pumping for a patient with fulminant myocarditis. Heart Vessels 27, 639-642.

Pollack, A., Kontorovich, A.R., Fuster, V., and Dec, G.W. (2015). Viral myocarditis-diagnosis, treatment options and current controversies. Nat Rev Cardiol 12, 670-680.

Puymirat, E., Fagon, J.Y., Aegerter, P., Diehl, J.L., Monnier, A., HauwBerlemont, C., Boissier, F., Chatellier, G., Guidet, B., Danchin, N., et al. (2017). Cardiogenic shock in intensive care units: evolution of prevalence, patient profile, management and outcomes, 1997-2012. Eur J Heart Fail 19, 192-200.

Rodrigues J.P.F., Caldas I.S., Goncalves R.V., Almeida L.A., Souza R.L. M., and Novaes R.D. (2017). S. mansoni-T. cruzi co-infection modulates arginase-1/iNOS expression, liver and heart disease in mice. Nitric Oxide 66, 43-52.

Rodríguez, A., Alvarez-Rocha, L., Sirvent, J.M., Zaragoza, R., Nieto, M., Arenzana, A., Luque, P., Socías, L., Martín, M., Navarro, D., et al. (2012). Recomendaciones del Grupo de Trabajo Enfermedades Infecciosas (GTEI) de la Sociedad Española de Medicina Intensiva, Crítica y Unidades Coronarias (SEMICYUC) y el Grupo de Estudio de Infecciones en el Paciente Crítico (GEIPC) de la Sociedad Española de Enfermedades Infecciosas y Microbiología clínica (SEIMC) para el diagnóstico y tratamiento de la gripe $\mathrm{A} / \mathrm{H} 1 \mathrm{~N} 1$ en pacientes adultos graves hospitalizados en las Unidades de Cuidados Intensivos. Med Intensiva 36, 103-137.

Section of Precision Medical of Chinese Society of Cardiology of Chinese Medical Association, Editorial Board of Chinese Journal of Cardiology, and Working Group on Adult Myocarditis. (2017). Chinese expert consensus statement on clinical diagnosis and treatment of fulminant myocarditis in adults. Zhonghua Xin Xue Guan Bing Za Zhi 45, 742752.

Shioji, K., Kishimoto, C., and Sasayama, S. (2001). Fc receptor-mediated inhibitory effect of immunoglobulin therapy on autoimmune giant cell myocarditis. Circ Res 89, 540-546.

Ting, M., Wang, C.H., Tsao, C.I., Huang, S.C., Chi, N.H., Chou, N.K., Chen, Y.S., and Wang, S.S. (2016). Heart transplantation under mechanical circulatory support for acute fulminant myocarditis with cardiogenic shock. Transplant Proc 48, 951-955.

Tschope C., Muller I., Xia Y., Savvatis K., Pappritz K., Pinkert S., Lassner D., Heimesaat M.M., Spillmann F., Miteva K., et al. (2017). NOD2 (nucleotide-binding oligomerization domain 2) is a major pathogenic mediator of Coxsackievirus B3-induced myocarditis. Circ Heart Fail 10, pii:e003870.

Ukimura, A., Izumi, T., Matsumori, A., and Clinical Research Committee on Myoc, A. (2010). A national survey on myocarditis associated with the 2009 influenza A (H1N1) pandemic in Japan. Circ J 74, 2193-2199.

Ukimura, A., Ooi, Y., Kanzaki, Y., Inomata, T., and Izumi, T. (2013). A national survey on myocarditis associated with influenza H1N1pdm2009 in the pandemic and postpandemic season in Japan. J Infect Chemother 19, 426-431.

Wang, D., Li, S., Jiang, J., Yan, J., Zhao, C., Wang, Y., Ma, Y., Zeng, H., Guo, X., Wang, H., et al. (2019). Chinese society of cardiology expert consensus statement on the diagnosis and treatment of adult fulminant myocarditis. Sci China Life Sci 62, 187-202.

Zhang, L., Wei, T.T., Li, Y., Li, J., Fan, Y., Huang, F.Q., Cai, Y.Y., Ma, G., Liu, J.F., Chen, Q.Q., et al. (2018). Functional metabolomics characterizes a key role for $N$-acetylneuraminic acid in coronary artery diseases. Circulation 137, 1374-1390. 


\section{SUPPORTING INFORMATION}

Figure S1 Myocardial fibrosis of differently treated A/J mice.

Table S1 The cardiac measures in four groups

The supporting information is available online at http://life.scichina.com and https://link.springer.com. The supporting materials are published as submitted, without typesetting or editing. The responsibility for scientific accuracy and content remains entirely with the authors. 